\title{
二氟甲基重氮甲烷作为含氟砌块的应用研究进展
}

\author{
朱文庆 $a$ 许婷怡 $a$ 韩文勇 $*, b, c$ \\ ( ${ }^{a}$ 西安工程大学环境与化学工程学院 西安 710048) \\ ( ${ }^{b}$ 遵义医科大学药学院 贵州省生物催化与手性药物合成重点实验室 贵州遵义 563006) \\ ( ${ }^{c}$ 遵义医科大学 基础药理教育部重点实验室暨特色民族药教育部国际合作联合实验室 贵州遵义 563006)
}

\begin{abstract}
摘要 二氟甲基化反应经过近二十年的发展已取得了较为显著的研究成果. 除直接和间接二氟甲基化反应外，二氟甲 基砌块参与的化学转化是一种向有机化合物中引入二氟甲基的新策略. 相比于亲电、亲核和自由基二氟甲基化试剂, 二氟甲基砌块的种类仍然有限并有待进一步开发. 其中, 二氟甲基重氮甲烷是近年来发展的一类新型含氟砌块, 受到 了研究学者们的高度关注. 综述了二氟甲基重氮甲烷及其稳定替代物的最新应用研究进展, 着重介绍了其反应类型及 相关机理，并对今后的发展方向进行了展望.
\end{abstract}

关键词 二氟甲基重氮甲烷; 二氟甲基化; 含氟砌块; 吡唑

\section{Recent Progress in the Application of Difluoromethyl Diazomethane as Fluorine-Containing Building Block}

\author{
Zhu, Wenqing $^{a} \quad \mathrm{Xu}$, Tingyi ${ }^{a} \quad$ Han, Wenyong ${ }^{*, b, c}$
}

( ${ }^{a}$ School of Environmental and Chemical Engineering, Xi'an Polytechnic University, Xi'an 710048)

$\left({ }^{b}\right.$ Key Laboratory of Biocatalysis \& Chiral Drug Synthesis of Guizhou Province, School of Pharmacy, Zunyi Medical University, Zunyi, Guizhou 563006)

( ${ }^{c}$ Key Laboratory of Basic Pharmacology of Ministry of Education and Joint International Research Laboratory of Ethnomedicine of Ministry of Education, Zunyi Medical University, Zunyi, Guizhou 563000)

\begin{abstract}
Difluoromethylation has achieved remarkable progress in the past two decades. In addition to the direct difluoromethylation and the stepwise difluoromethylation, chemical transformation starting from $\mathrm{CF}_{2} \mathrm{H}$-containing building blocks is a novel strategy to introduce difluoromethyl group into organic compounds. Compared with electrophilic-, nucleophilic- and free radical-difluoromethylation reagents, the types of $\mathrm{CF}_{2} \mathrm{H}$-containing building blocks are still limited and less explored. Among them, difluoromethyl diazomethane $\left(\mathrm{CF}_{2} \mathrm{HCHN}_{2}\right)$ is a new type of fluorine-containing building block developed in recent years, which has received much attention from researchers. The recent progress in the application of difluoromethyl diazomethane and its surrogates as fluorine-containing building blocks is summarized, focusing on the reaction types and related mechanisms, and the developmental direction in the future is prospected.
\end{abstract}

Keywords difluoromethyl diazomethane; difluoromethylation; fluorinated building block; pyrazole

氟原子或含氟基团的引入所产生的诱导效应、伪拟 效应、阻断效应、亲脂性效应及氢键效应赋予含氟化合 物独特的性质. 氟原子的强电负性和碳氟键键能较高的 特性可用于改善化合物的代谢稳定性、物理性质、生物 利用度、配体-受体间的相互作用及选择性，这决定了其 在生物医药领域的巨大潜力和科研价值 ${ }^{[1]}$.二氟甲基
$\left(\mathrm{CF}_{2} \mathrm{H}\right)$ 由于含有一个弱酸化的 $\mathrm{C}-\mathrm{H}$ 键，是一种较为特 殊的含氟基团，可作为羟基、颈基及羟甲基的电子等排 体，能够调节分子的生物活性和代谢稳定性等 ${ }^{[2]}$, 在医 药、农药等领域起着与其他氟烷基同样重要的作用(图 $1)^{[3]}$.

\footnotetext{
* Corresponding authors. E-mail: hanwy@zmu.edu.cn; hanwy@126.com

Received September 28, 2020; revised November 2, 2020; published online November 28, 2020.

Project supported by the National Natural Science Foundation of China (No. 21762054) and the Outstanding Youth Talent Support Programs from Zunyi Medical University (No. 18ZY-002).

国家自然科学基金(No. 21762054)和遵义医科大学优秀青年人才支持计划(No. 18ZY-002)资助项目.
} 
<smiles>Cc1cc(O)c(C(=O)C2CC2C(F)(F)F)c(=O)o1</smiles><smiles>COc1ccc(-c2cc(C(F)F)nn2-c2ccc(S(N)(=O)=O)cc2)cc1F</smiles>

Deracoxib<smiles>O=C(Nc1c(Cl)cncc1Cl)c1ccc(OC(F)(F)F)c(OCC2CC2)c1</smiles>

Roflumilast

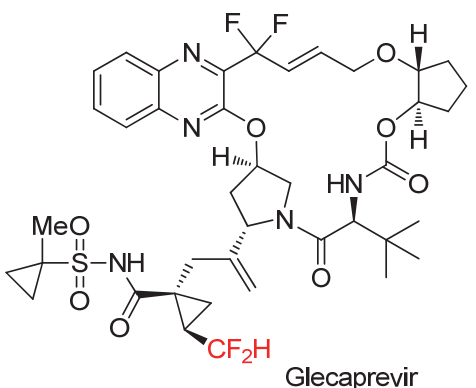<smiles>CCCC1CC2CCC1c1cccc(NC(=O)c3cn(C)nc3C(F)F)c12</smiles>

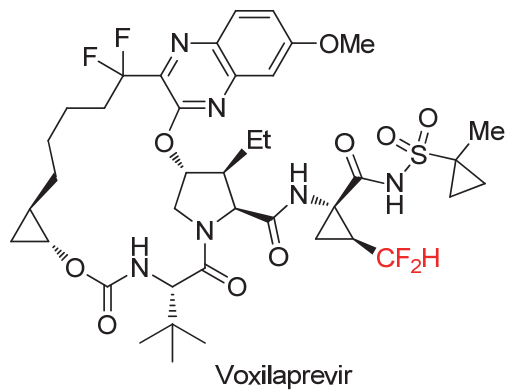

图 1 含有二氟甲基的药物和生物活性分子

Figure 1 Difluoromethyl-containing drugs and bioactive molecules

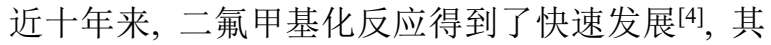
中 “直接” 二氟甲基化和 “间接” 二氟甲基化反应已被 广泛研究. 研究者开发了许多不同类型的亲电、亲核及 自由基二氟甲基试剂, 如 $\mathrm{Ph}_{3} \mathrm{P}^{+} \mathrm{CF}_{2} \mathrm{CO}_{2}^{-}$(PDFA) ${ }^{[5]}$ 、 $\mathrm{TMSCF}_{2} \mathrm{Br}^{[6]} 、 \mathrm{BrCF}_{2} \mathrm{H}^{[7]} 、 \mathrm{TMSCF}_{2} \mathrm{H}^{[8]}$ 、二氟甲基杂芳 基砜[9]、(DMPU) ${ }_{2} \mathrm{Zn}\left(\mathrm{CF}_{2} \mathrm{H}\right)_{2}{ }^{[10]}$ 和 $\left[(\mathrm{SIPr}) \mathrm{Ag}-\left(\mathrm{CF}_{2} \mathrm{H}\right)\right]^{[11]}$ 等. 除此之外, 含二氟甲基砌块参与的化学转化也被用于复 杂含二氟甲基化合物的构建. 然而, 目前含二氟甲基砌 块的类型较少, 主要包括 3-溴-3,3-二氟丙烯 ${ }^{[12]} 、 2-\mathrm{CF}_{2} \mathrm{H}-$ $N-\mathrm{Ts}$ 氮杂环丙烷 ${ }^{[13]}$ 和溴二氟甲基膦酸二乙酯等 ${ }^{[14]}$, 导 致基于含氟砌块参与的化学反应构建二氟甲基取代的 复杂有机化合物的方法发展较慢. 因此, 开发其它类型 的含氟砌块用于二氟甲基化合物的快速合成显得十分 必要.

重氮化合物是一类高活性的多功能试剂, 因其独特 的结构可以参与多种有机化学反应, 从而构建不同类型 的功能分子 ${ }^{[15]} .1943$ 年, Gilman 和 Jones ${ }^{[16]}$ 首次报道三氟 乙胺盐酸盐(1)在亚硝酸钠酸性水溶液中可以生成一种 重要的三氟甲基砌块一一三氟甲基重氮甲烷 $(2$, $\left.\mathrm{CF}_{3} \mathrm{CHN}_{2}\right)$ (Scheme 1, a). 三氟甲基重氮甲烷(2)是一种 低沸点的黄色液体 (沸点为 $13{ }^{\circ} \mathrm{C}$ ), 一般溶解在有机溶 剂中低温保存使用, 稳定性较好. 经过近 80 年的发展, 该试剂可作为 1,3-偶极子与烯烃、炔烃、联烯及苯炔等 发生 $[3+2]$ 环加成反应, 与甘氨酸酯亚胺发生 $[3+3]$ 环 加成反应. 此外, 2 还能作为 $N$-亲电试剂、 $C$-亲核试剂和 卡宾前体参与多种有机反应, 被广泛用于各种含三氟甲 基化合物的合成 ${ }^{[17]}$.

尽管只存在一个氟原子的差异, 二氟甲基重氮甲烷 $\left(4, \mathrm{CF}_{2} \mathrm{HCHN}_{2}\right)$ 的合成显得困难重重 ${ }^{[18]}$. 直到 2015 年,
Mykhailiuk $^{[19]}$ 才首次报道了该重要二氟甲基砌块的制 备: 以二氟乙胺 $(\mathbf{3})$ 和亚硝酸叔丁酯为原料，冰醋酸为催 化剂, 在氯仿中加热回流 $10 \mathrm{~min}$ 后, 可原位得到亮黄色 二氟甲基重氮甲烷(4)的氯仿溶液(Scheme 1, b). 相比于 已被深入研究的三氟甲基重氮甲烷，二氟甲基重氮甲烷 活性较高, 但稳定性较差, 一般通过原位制备或利用连 续流动方法来进行后续转化. 导致两者性质差异的主要 原因可能是: (1)立体位阻较大的三氟甲基基团屏蔽了重 氮活性位点，导致三氟甲基重氮甲烷的反应活性较低； (2)诱导效应稳定了三氟甲基重氮甲烷的共振式 2 "'和二 氟甲基重氮甲烷的共振式 4"', 由于三氟甲基的电负性 比二氟甲基大，从而导致三氟甲基重氮甲烷较二氟甲基 重氮甲烷稳定(Scheme 1, c). 二氟甲基重氮甲烷较差的 稳定性也极大地限制了它的反应类型. 本文对二氟甲基 重氮甲烷作为含氟砌块的应用进行了归纳总结, 重点讨 论了其反应类型，主要包括 1,3-偶极环加成反应、金属 卡宾反应、酯化反应和偕双官能化反应，同时介绍了二 氟甲基重氮甲烷的稳定替代物. 最后，也对二氟甲基重 氮甲烷的今后应用方向作了展望.

\section{1,3-偶极环加成反应}

2015 年, Mykhailiuk ${ }^{[19]}$ 通过二氟甲基重氮甲烷(4)与 炔烃的 $[3+2]$ 环加成反应成功合成了二氟甲基取代的吡 唑类化合物 5, 并实现了产物的克级制备(Scheme 2). 反 应无需使用惰性气体保护, 在室温下利用原位生成的二 氟甲基重氮甲烷(4)即可发生 1,3-偶极环加成反应. 考察 底物普适范围发现，吸电子基团有利于反应的进行，而 供电子基团会抑制反应的发生. 
(a) Preparation of $\mathrm{CF}_{3} \mathrm{CHN}_{2}$ (2)

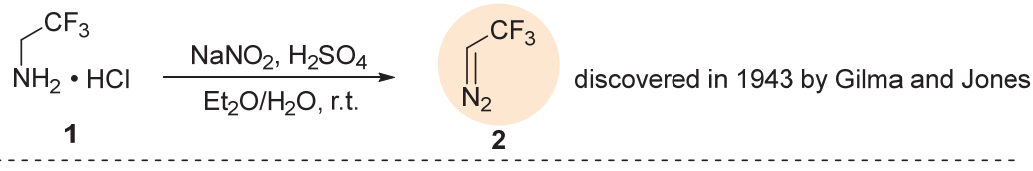

(b) Preparation of $\mathrm{CF}_{2} \mathrm{HCHN}_{2}$ (4)

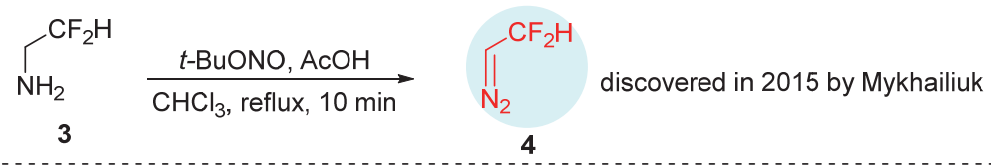

(c) Properties of $\mathrm{CF}_{3} \mathrm{CHN}_{2}$ (2) and $\mathrm{CF}_{2} \mathrm{HCHN}_{2}$ (4)
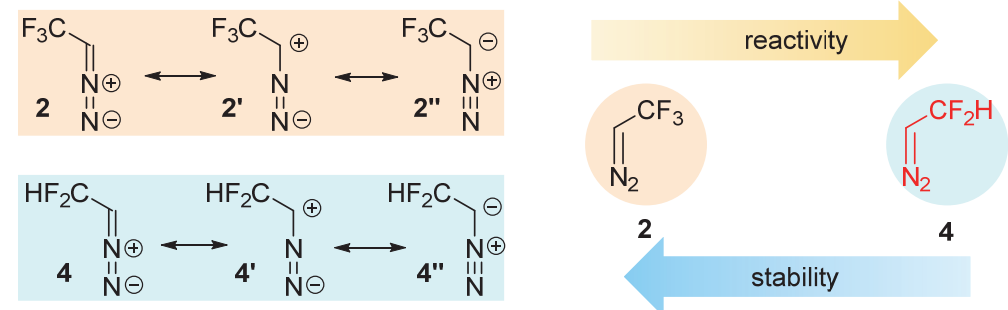

图式 1 三氟甲基重氮甲烷(2)和二氟甲基重氮甲烷(4)的制备及其性质

Scheme 1 Preparations of $\mathrm{CF}_{3} \mathrm{CHN}_{2}$ (2) and $\mathrm{CF}_{2} \mathrm{HCHN}_{2}$ (4) as well as their properties

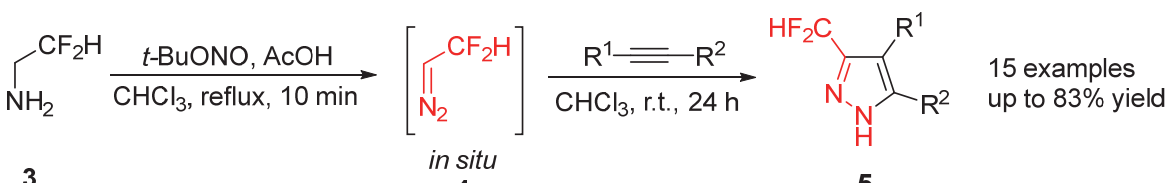

3

4

图式 2 二氟甲基重氮甲烷与缺电子炔烃的 $[3+2]$ 环加成反应

Scheme $2[3+2]$ Cycloaddition reaction of $\mathrm{CF}_{2} \mathrm{HCHN}_{2}$ with electron-deficient alkynes

然而由于商业易得的缺电子炔烃种类较少, 上述方 案的应用范围有限. 因此, 研究学者们将目光转向了缺 电子烯烃, 并就二氟甲基重氮甲烷(4)与不同类型烯烃 的反应性展开了系统研究.

2017 年, 吴勇和 Mykhailiuk 团队[20]共同报道了原 位生成的二氟甲基重氮甲烷(4)与单取代缺电子烯烃和
$N$-芳基马来酰亚胺的 $[3+2]$ 环加成反应，得到了一类二 氟甲基取代的吡唑啉化合物 6 和 8 (Scheme 3). 值得一 提的是, 6 经氧化可转化为 $\mathbf{5}$, 以 $N$-芳基马来酰亚胺为底 物的反应得到的 3,5-二取代吡唑啉类化合物 7 经柱层析 分离、在酸性条件下或在 DMSO- $d_{6}$ 中均会转化为 $\mathbf{8}$ (Scheme 3). 2018 年, 该团队 ${ }^{[21]}$ 在此研究基础上, 通过加

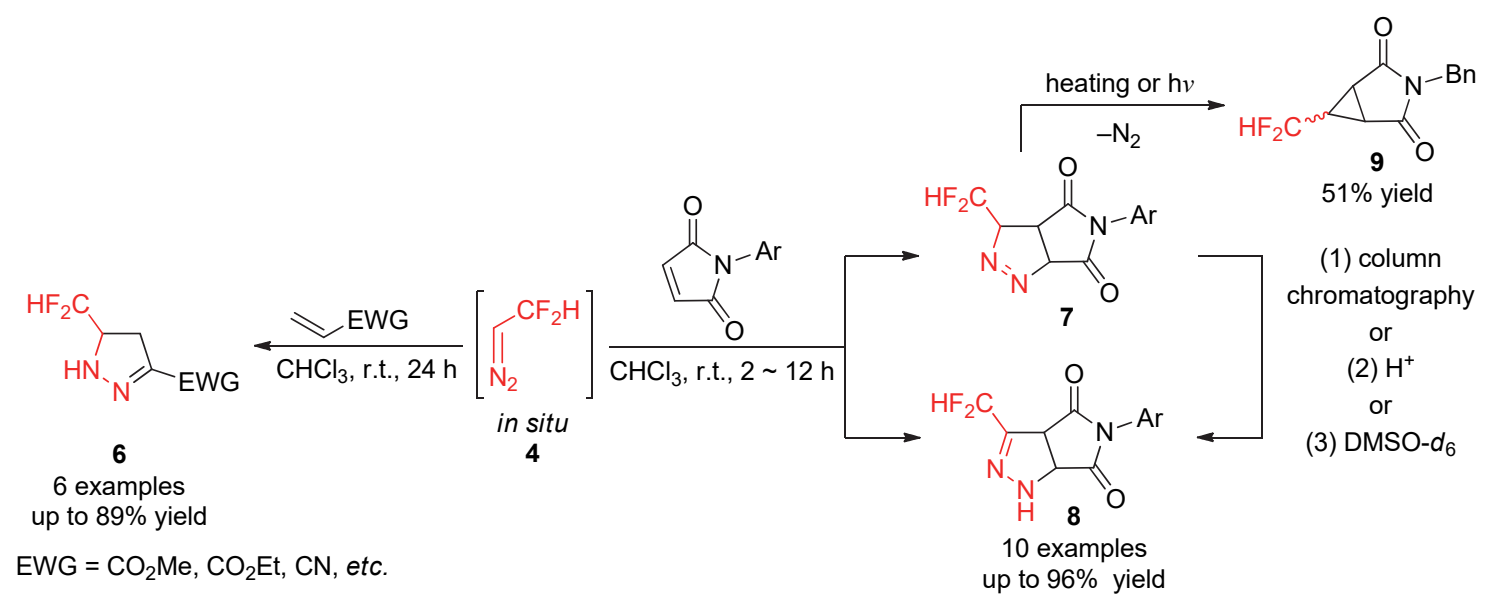

图式 3 二氟甲基重氮甲烷与缺电子烯烃的 $[3+2]$ 环加成反应

Scheme $3[3+2]$ cycloaddition reaction of $\mathrm{CF}_{2} \mathrm{HCHN}_{2}$ with electron-deficient alkenes 
热或光化学辐射逐步裂解吡唑啉中的 $\mathrm{C}-\mathrm{N}=\mathrm{N}-\mathrm{C}$ 键 使其重新构建成环丙烷，实现了 3-茮基-6-二氟甲基-3氮杂双环[3.1.0]己烷-2,4-二酮(9)的合成(Scheme 3).

2017 年, Mykhailiuk 课题组 ${ }^{[22]}$ 报道了二氟甲基重氮 甲烷(4)与缺电子烯烃及亚硝酸叔丁酯 $(t-\mathrm{BuONO})$ 的 $[3+$ 2]环加成 $/ N$-亚硝化反应, 得到了 $N$-亚硝基取代的二氟 甲基吡唑啉类化合物 11 (Scheme 4). 在初始条件下, 反 应得到 10 和 11 的混合物, 控制实验结果表明, 10 与未 消耗完全的亚硝酸叔丁酯反应转化为 11. 由此, 作者确 定了一锅两步法的合成路线，以高达 $67 \%$ 的产率得到了 18 个同时含有二氟甲基和亚硝基的吡唑啉 11. 除此之 外, 产物在 $\mathrm{MnO}_{2}$ 氧化作用下可进一步转化为二氟甲基 取代吡唑类化合物 12, 其在高温下则转化为异噁唑啉 类化合物 13.

2017 年, Cossy、Mykhailiuk 等[23]报道了通过乙炔衍 生物、 $\mathrm{TMSCF}_{3}$ 和重氮化合物的 $[2+1] /[3+2]$ 环加成反 应合成 4-氟哒嗪的路线(Scheme 5). 在该反应体系中, 苯乙炔 $(14)$ 与 $\mathrm{TMSCF}_{3}$ 经 $[2+1]$ 环加成反应得到 1-苯基3,3-二氟环丙烯(15), 再与二氟甲基重氮甲烷(4)发生 $[3+2]$ 环加成反应，最终得到 4-氟-6-二氟甲基哒嗪(16). 产物经芳香亲核取代反应 $\left(\mathrm{S}_{\mathrm{N}} \mathrm{Ar}\right)$ 可进一步转化成 3,4,6三取代的哒嗪类化合物. 与三氟甲基重氮甲烷(2)为底 物的反应相比，二氟甲基重氮甲烷(4)参与的反应所需 反应时间更长, 相应的产率更低, 且底物普适范围有待
进一步考察.

我们研究小组自 2017 年起报道了一系列 3-烯基氧 化吲哚 17 与原位生成的二氟甲基重氮甲烷(4)的环加成 反应(Scheme 6), 发现通过改变溶剂可以获得不同类型 的产物：(1)在二氯甲烷(DCM)中，经过 $[3+2]$ 环加成反 应以高产率、高非对映选择性获得了二氟甲基取代的螺 环氧化吲哚吡唑类化合物 $\mathbf{1 8}^{[24]}$; (2)在甲苯中回流 $2 \mathrm{~h}$ 后, 18 可进一步脱去一分子氮气转化为含有二氟甲基的螺 环丙烷氧化吲哚类化合物 19 , 值得注意的是, 该类化合 物对前列腺癌(PC-3)和人非小细胞肺癌细胞(A549)的生 长有一定的抑制作用，且该方法可用于合成一种 HIV-1 NNRTI 抑制剂类似物 ${ }^{[25]}$; (3)以四氢呋喃(THF)为溶剂时, 经[3+2]环加成和 1,3-H 迁移过程, 可得到螺[氧化吲哚3,3'-吡唑]-5'二氟甲基-4'-甲酸酯类化合物 $\mathbf{2 0}^{\text {[26]; (4)以乙 }}$ 醚为溶剂时, 在氧气氛围下, 经 $[3+2]$ 环加成/1,3-H 迁 移/重排/脱氢过程，可制备二氟甲基取代的吡唑并 [1,5-c]喹唑啉类化合物 21. 该反应可以放大到克级，且 产物只需经简单的过滤、洗涤和干燥过程实现分离纯 化 ${ }^{[27]}$.

在此基础上，我们设想原位生成的二氟甲基重氮甲 烷(4)是否可以与 $\alpha, \beta$-不饱和硝基烯烃 22 经历[3+2]环 加成/1,3-H 迁移/脱氢的串联反应过程得到 3-(二氟甲 基)- $1 H$-吡唑 23 , 并进一步转化为其它类型的药物分子 (Scheme 7, a). 在实施该反应过程中, 我们并未发现预

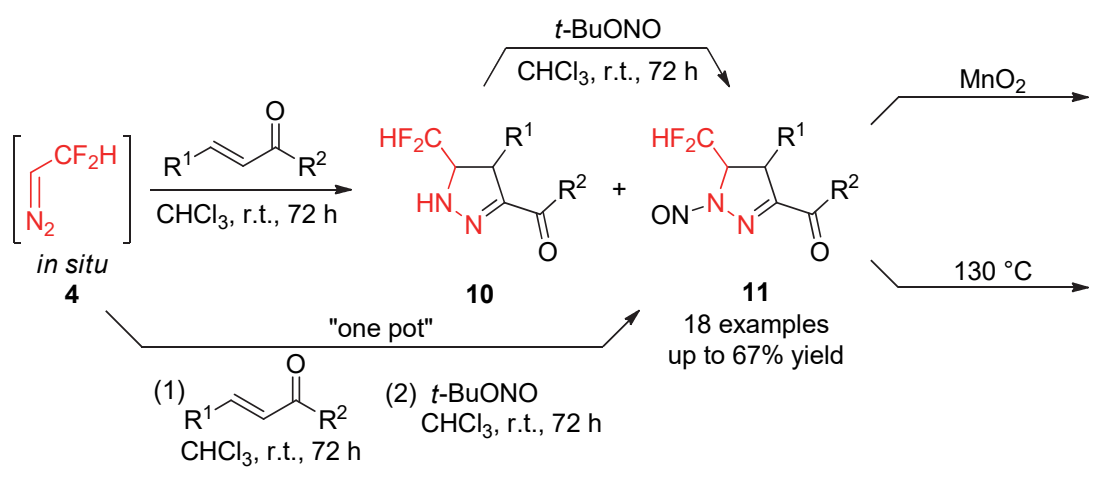

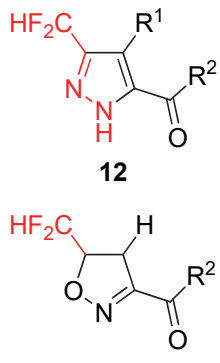

13

图式 4 利用二氟甲基重氮甲烷合成 $N$-亚硝基二氟甲基吡唑啉类化合物

Scheme 4 Synthesis of $N$-nitroso $\mathrm{CF}_{2} \mathrm{H}$-pyrazolines with $\mathrm{CF}_{2} \mathrm{HCHN}_{2}$

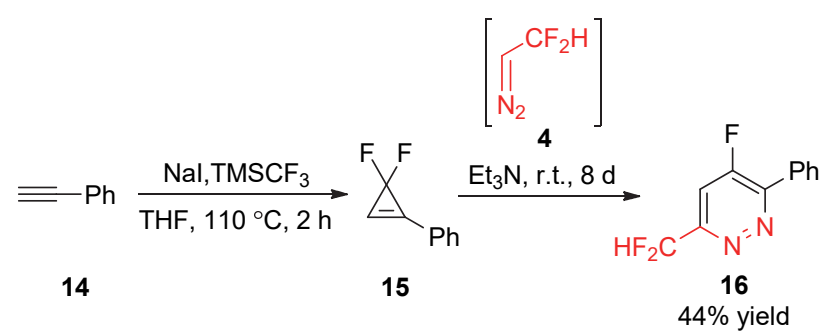

图式 5 利用二氟甲基重氮甲烷合成 4-氟-6-二氟甲基哒嗪

Scheme 5 Synthesis of 4-fluoro-6-(difluoromethyl)pyridazine with $\mathrm{CF}_{2} \mathrm{HCHN}_{2}$ 


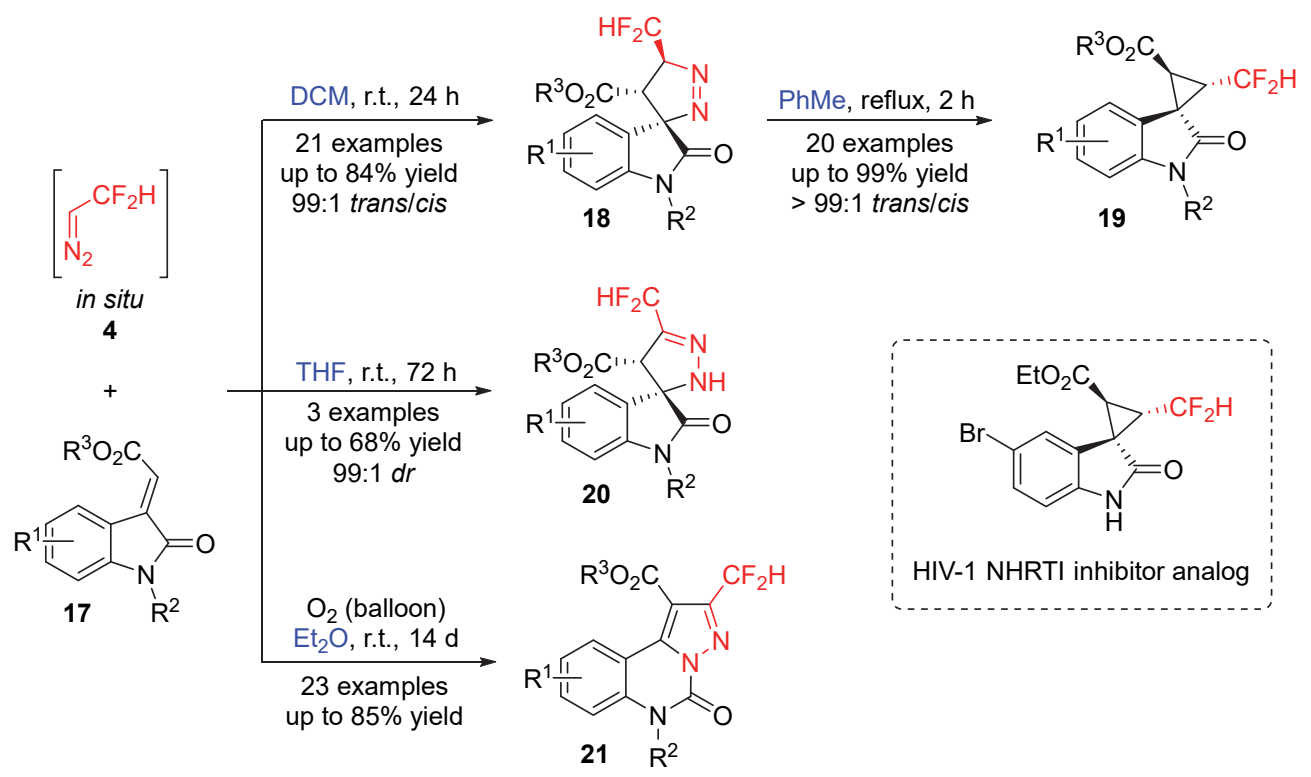

图式 6 二氟甲基重氮甲烷在含氮杂环化合物合成中的应用

Scheme 6 Application of $\mathrm{CF}_{2} \mathrm{HCHN}_{2}$ in the synthesis of $\mathrm{N}$-heterocyclics

(a) Synthesis design of 3-(difluoromethyl)-1H-pyrazoles

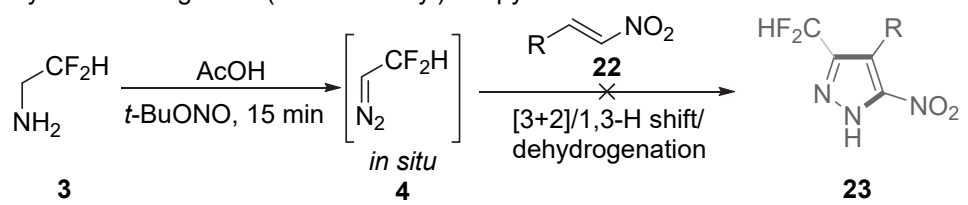

(b) Unexpected aza-Michael addition $/ N$-nitrosation process

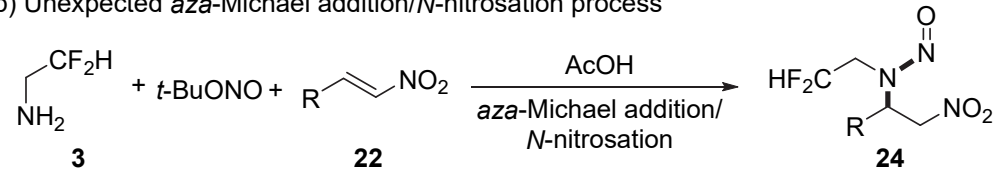

(c) [3+2] Cycloaddition with ex situ generated $\mathrm{CF}_{2} \mathrm{HCHN}_{2}$

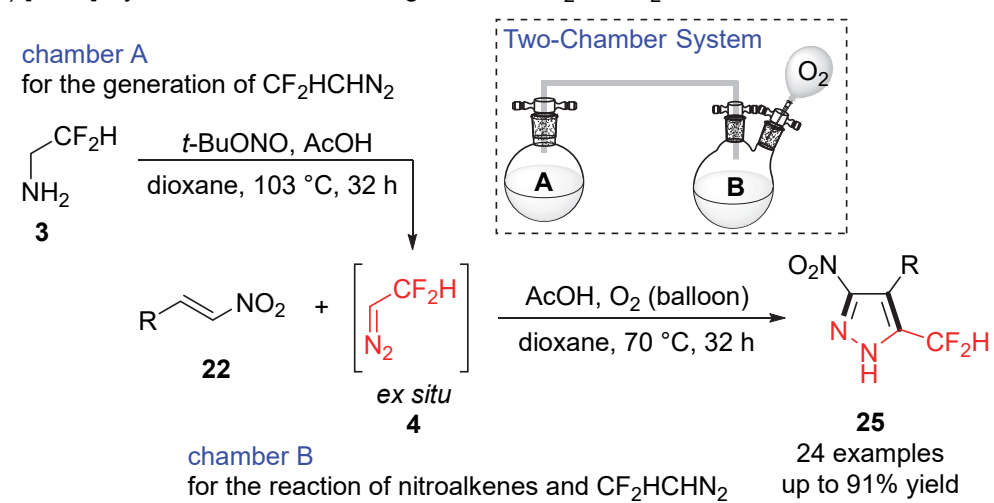

图式 7 不期望的三组分反应以及二氟甲基吡唑类化合物的合成设计

Scheme 7 Unexpected three-component reaction as well as synthetic design of $\mathrm{CF}_{2} \mathrm{H}$-containing pyrazoles

期产物 23 的生成, 而得到了一类含二氟乙基的 $N$-亚硝 基胺类化合物 24 (Scheme 7, b) ${ }^{[28]}$. 后续的反应机理研究 表明 24 的生成是由于二氟乙胺(3)与硝基烯烃 22 发生 $a z a-$ Michael 加成反应的速率远大于原位生成二氟甲基 重氮甲烷(4)的速率. 为避免反应过程中存在的竞争反
应( $a z a-$-Michael 加成反应)的发生, 我们重新设计了以非 原位生成的二氟甲基重氮甲烷(4)为原料的合成路线, 采用“双瓶”体系, 在氧气氛围下, 经[3+2]环加成/1,3-H 迁移/氧化芳构化串联过程, 成功实现了二氟甲基取代 的吡唑类化合物 25 的合成(Scheme 7, c) ${ }^{[29]}$. 


\section{2 金属卡宾反应}

由于反应条件苛刻、合成步骤多且操作复杂、底物 普适性差, 氟烷基取代异噁唑类化合物的合成一直以来 受到较大限制. 为解决这一问题, 胡祥国课题组 ${ }^{[30]}$ 在 2018 年设计了一种在铜催化下 “一锅法” 制备 3-二氟 甲基异噁唑类化合物 27 的合成路线(Scheme 8). 该方法 原料易得, 产率高且底物普适性好, 更重要的是, 不需 要处理不稳定或危险的中间体. 通过控制实验, 作者推 测反应经历了铜催化的串联反应: 原位生成的二氟甲基 重氮甲烷(4)与 $\mathrm{Cu}(\mathrm{I})$ 形成铜卡宾 $\mathbf{A}$, 接着与端炔锌试剂 B 反应发生转移金属化和迁移插入生成铜物种 $\mathbf{C}$, 铜物 种 $\mathbf{C}$ 被亚硝基离子捕获后进一步转化为 $\mathbf{D}$, 经重排和 5-endo-dig 环化反应后得到目标产物 27.
次年，该课题组 ${ }^{[31]}$ 发展了铜催化的羧酸 28、腈 29、 二氟乙胺 $(3)$ 和亚硝酸叔丁酯的四组分反应，通过原位 生成的二氟甲基重氮甲烷(4)参与该反应，以高达 $91 \%$ 的收率得到一系列 $N$-二氟乙基酰亚胺 30 (Scheme 9). 反应底物普适性较好，不同取代基的芳香羧酸与腈类化 合物都能以中高产率获得相应产物。研究表明，实现该 反应的关键在于通过高温及低浓度的反应条件抑制羧 酸 28 与二氟甲基重氮甲烷(4)的酯化反应(Scheme 9, Path B). 作者认为该反应的机理是 4 与铜催化剂反应形 成铜卡宾 $\mathbf{A}$ ，进一步被乙腈进攻后得到中间体 $\mathbf{B}$ ，再经 羧酸 28 亲核加成、Mumm 重排得到二氟乙基化的酰亚 胺 30. 此外, 该方法还适用于药物的后期官能化修饰, 如赤霉酸、莽草酸和胆酸, 这对开发新型酰亚胺类药物 分子具有重要价值(图 2).

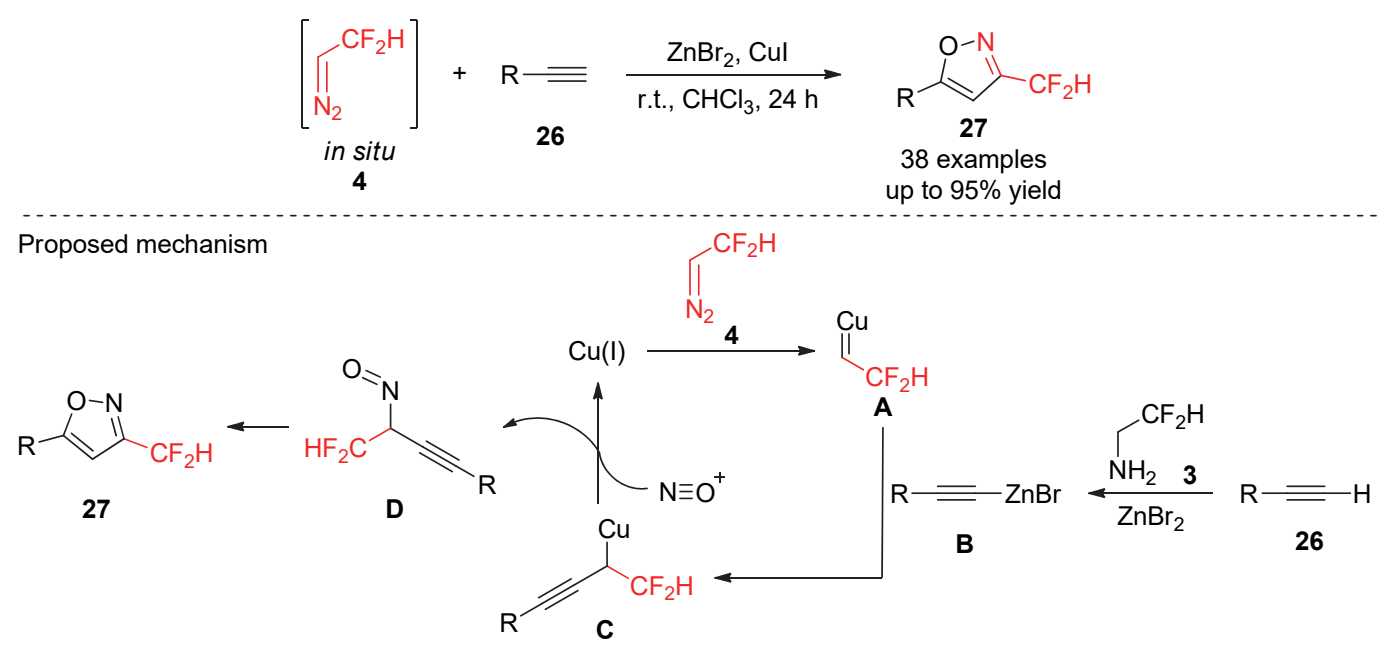

图式 8 利用二氟甲基重氮甲烷合成二氟甲基取代异噁唑类化合物

Scheme 8 Synthesis of difluoromethyl substituted isoxazole compounds with $\mathrm{CF}_{2} \mathrm{HCHN}_{2}$

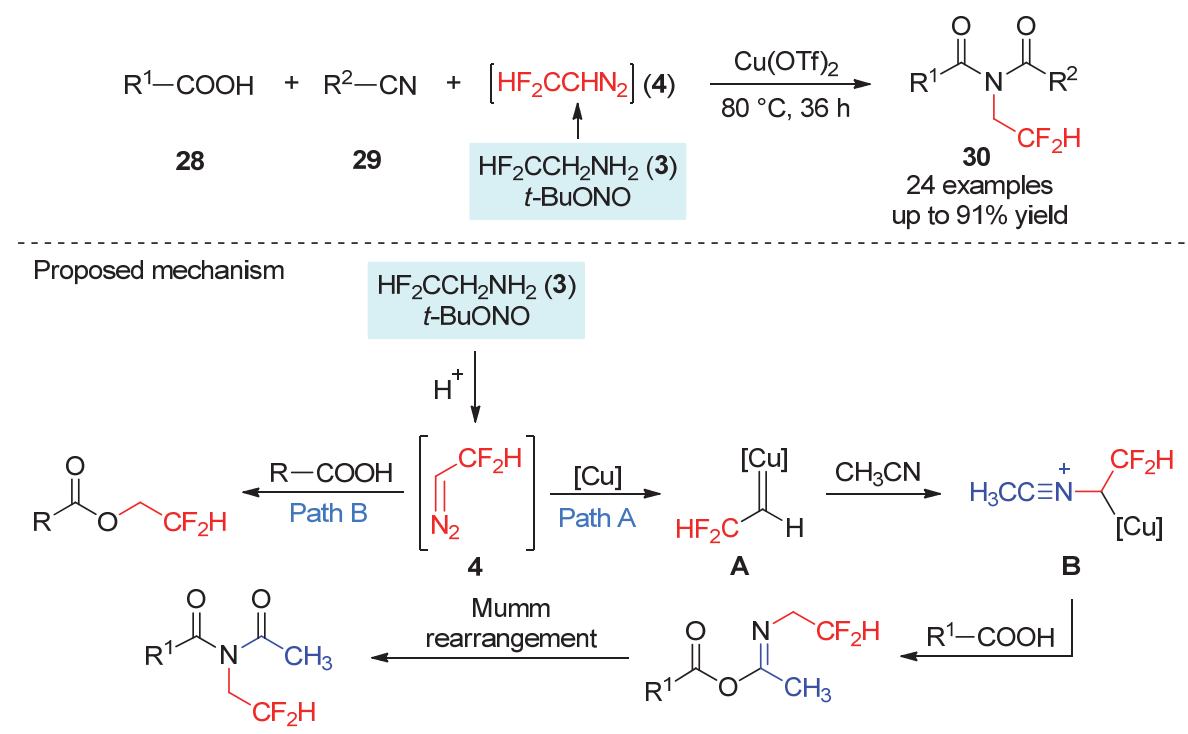

图式 9 铜催化四组分反应合成 $N$-二氟乙基酰亚胺类化合物

Scheme 9 Copper-catalyzed four-component reaction for the synthesis of $N$-difluoroethyl imides 


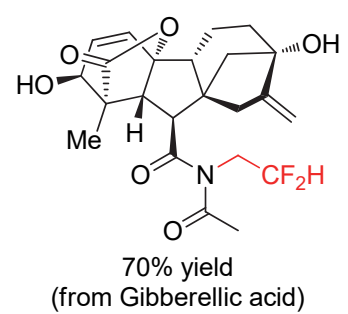

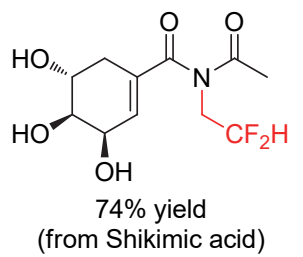

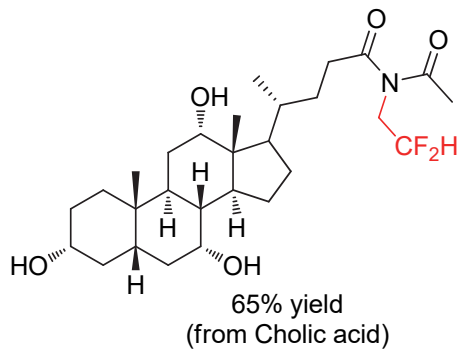

图 2 含 $N$-二氟乙基酰亚胺的药物分子类似物

Figure 2 Analogues of drug molecular containing $N$-difluoroethyl imides

\section{3 酯化反应}

2017 年, Mykhailiuk 等 ${ }^{[32]}$ 利用原位生成的二氟甲基 重氮甲烷(4)在其它极性官能团存在下选择性地对羧酸 31 进行酯化反应(Scheme 10, a). 该反应操作简便, 条件 温和, 无需催化剂. 重要的是, 该方法可实现多肽和蛋 白质等生物大分子的后期官能化修饰, 所得产物可作为 生物分子中的 ${ }^{19} \mathrm{~F}$ 探针, 进而通过扩散 ${ }^{19} \mathrm{~F}$ 核磁共振实验 确定分子大小或测量分子量.

同年, 胡祥国课题组 ${ }^{[33]}$ 对二氟甲基重氮甲烷(4)与 羧酸 31 的酯化反应进行了系统研究(Scheme 10, b). 不 同于 Mykhailiuk 等 ${ }^{[32]}$ 报道的一锅两步法的酯化反应，该 反应无需单独制备二氟甲基重氮甲烷(4), 产率高且底 物谱范围广, 反应速率根据取代基位点不同略有差别, 供电子基团会降低反应速率. 有趣的是, 将二氟乙胺替 换为三氟乙胺时, 未得到酯化反应产物, 而是通过 Mumm 反应生成了 $N$-三氟乙基亚胺. 据密度泛函理论 (DFT)计算结果推测, 三氟甲基重氮甲烷(2)与二氟甲基 重氮甲烷(4)的反应活性差异可能是由氟的电负性引起 的静电质力和吸引力所致. 鉴于酯基及含氟基团的引入 可以改善药物分子的性质，作者将该方法应用于药物、 天然产物的后期功能化修饰, 底物普适性较好且能够实 现克级反应(图 3).

\section{4 偕双官能化反应}

非共轭重氮化合物高度的反应性及不稳定性使其 在偕双官能化反应中的应用少见报道. 2019 年, 胡文浩 课题组 ${ }^{[34]}$ 在这一领域取得了进展. 他们利用二硒化物/ $N$-氟代双苯磺酰胺(NFSI) 体系的独特性质, 成功实现

(a)

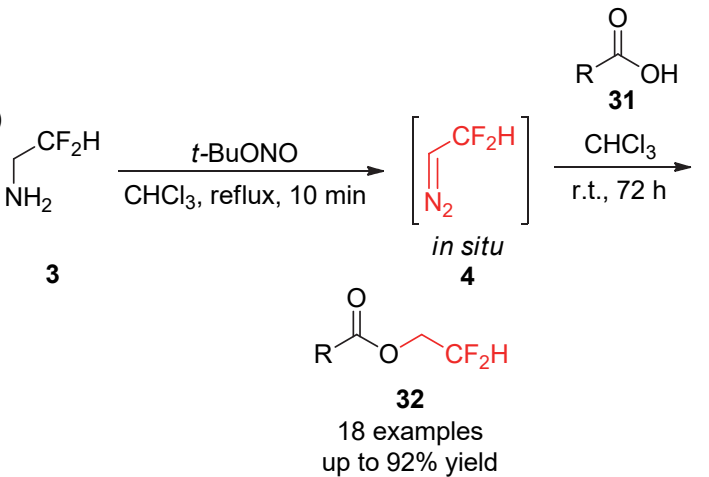

(b)

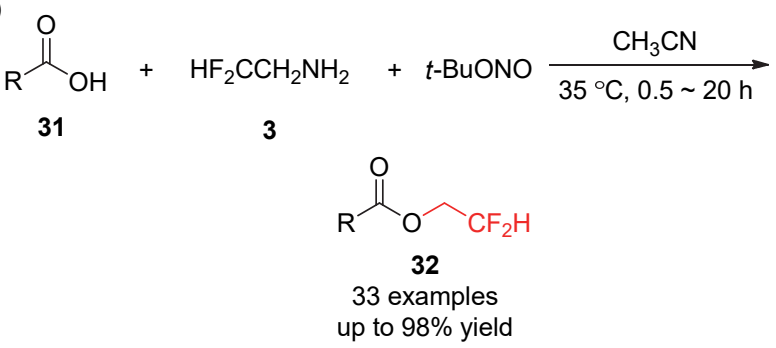

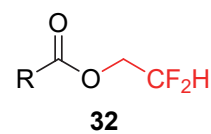

图式 10 二氟甲基重氮甲烷与羧酸的酯化反应

Scheme 10 Esterification of $\mathrm{CF}_{2} \mathrm{HCHN}_{2}$ with carboxylic acids<smiles>CC(C)(Oc1ccc(Cl)cc1)C(=O)OCC(F)(F)F</smiles><smiles>CC(C)(Oc1ccc(C(=O)c2ccc(Cl)cc2)cc1)C(=O)OCC(F)F</smiles>

$88 \%$ yield (from Fenofibric acid)<smiles>CCOC(=O)CC1(CC(=O)OCC(F)F)OCCc2c1[nH]c1c(CC)cccc21</smiles>

$76 \%$ yield (from Etodolac)<smiles>COc1ccc2c(c1)c(CC(=O)OCC(F)F)c(C)n2C(=O)c1ccc(Cl)cc1</smiles>

$86 \%$ yield (from Indometacin)<smiles>CCC(CC)O[C@H]1C=C(C(=O)OCC(F)F)C[C@H](NC(C)=O)[C@H]1NC(C)=O</smiles>

$86 \%$ yield (from Oseltamivir)

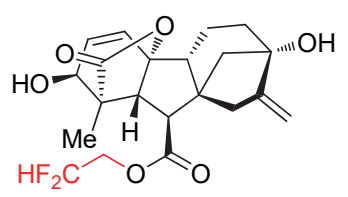

$73 \%$ yield (from Gibberellic acid)
图 3 市售药物和天然产物的二氟乙酯类似物 Figure 3 Difluoroethyl ester analogues of marketed drugs and natural products 
了包括二氟甲基重氮甲烷(4)在内的不同类型的非共轭 重氮化合物与二硒化物 33 的偕双官能化反应, 以高至 优秀的产率得到一系列含氟烷基取代基的偕氨基硒化 和磺酰化产物 35 (Scheme 11). 该方法同样适用于二硫 化物和二磅化物. 作者发现, 实现该反应的关键在于抑 制反应过程中非共轭重氮化合物的分解. 机理研究表 明, 该反应不受自由基抑制剂的影响，可能的反应路径 是二硒化物与 NFSI 反应形成硒酰亚胺中间体 $\mathbf{A}$, 接着 二氟甲基重氮甲烷(4)通过 $\mathrm{S}_{\mathrm{N}} 2$ 型亲核进攻中间体 $\mathbf{A}$ 中 带正电荷的硒原子, 随后发生分子内亲核进攻和脱氮气 过程生成相应的三组分产物 $\mathbf{3 5}$.

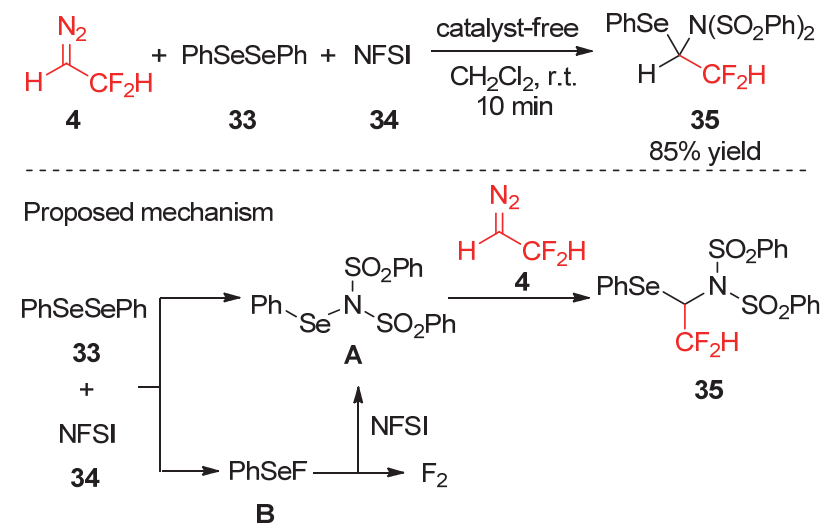

图式 11 二氟甲基重氮甲烷的偕双官能团化反应

Scheme 11 gem-Difunctionalization of $\mathrm{CF}_{2} \mathrm{HCHN}_{2}$

\section{5 连续流化学}

二氟甲基重氮甲烷(4)常态下为气体, 易挥发, 易分 解, 作为一种小分子重氮甲烷还具有潜在的毒性及爆炸 性, 在进行大规模合成和克量级反应时需要采取严格的 安全预防措施. 这些缺点使其在有机合成中的应用存在 很大的安全风险和局限性. 因此, 在 Mykhailiuk ${ }^{[19]}$ 的首 次报道不久, Koenigs 等 ${ }^{[35]}$ 开发了一种安全可靠的连续 流法以相同的原料实现了二氟甲基重氮甲烷(4)的合成, 并报道了其与不同亲偶极体的 $[3+2]$ 环加成反应 (Scheme 12). 反应以优秀的产率合成了一系列二氟甲 基取代的吡唑啉和吡唑类化合物 36. 值得一提的是, 作 者通过该方法得到了广泛存在于农用化学品的双氟烷 基取代吡唑，同时开辟了一条二氟甲基取代的磺化吡唑 啉的高效、原子经济的合成途径.

由于二氟甲基环丙烷结构单元存在于一些重要的 药物分子中, 且其合成方法报道较少, 2016 年, Koenigs 团队 ${ }^{[36]}$ 发展了二氟乙基铑卡宾参与的环丙烷化反应. 在采用相转移策略和使用原位生成的二氟甲基重氮甲 烷(4)均未获得理想产物的情况下, 作者基于利用连续 流反应器实现 $[3+2]$ 环加成反应的成功经验 ${ }^{[35]}$, 最终通 过连续流反应首次实现了铑催化二氟甲基取代环丙烷

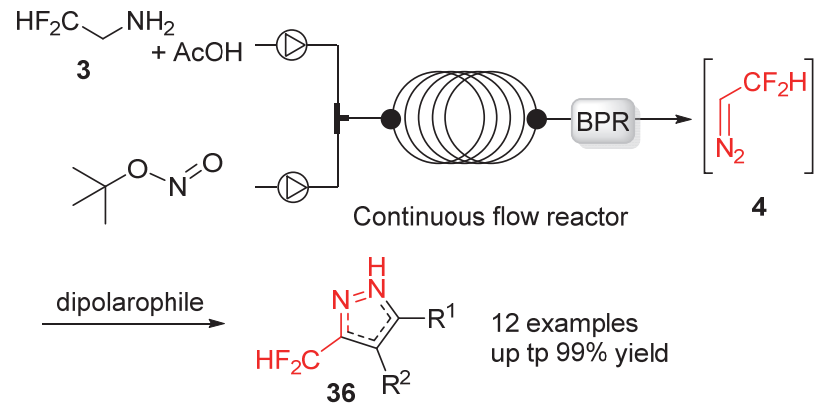

图式 12 连续流反应合成二氟甲基取代的吡唑啉和吡唑类化 合物

Scheme 12 Synthesis of $\mathrm{CF}_{2} \mathrm{H}$-containing pyrazolines and pyrazoles by continuous flow reaction

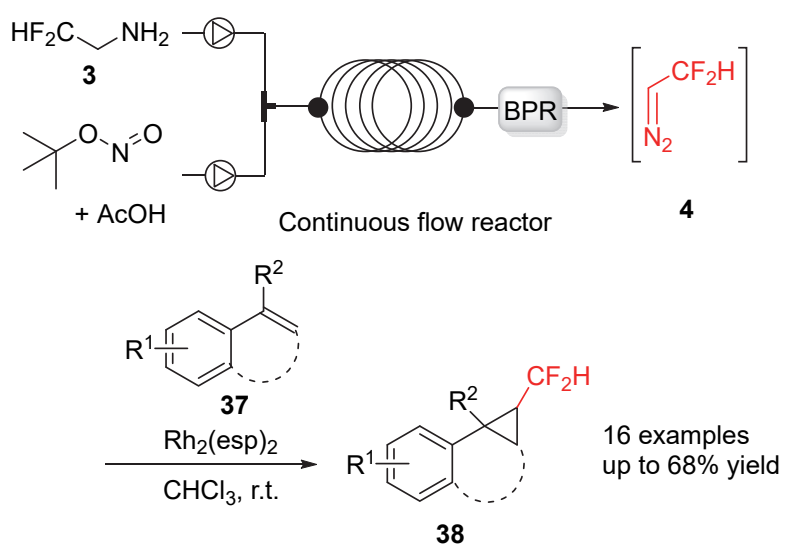

图式 13 连续流反应合成二氟甲基取代环丙烷 Scheme 13 Synthesis of difluoromethyl substituted cyclopropane by continuous flow reaction

38 的合成(Scheme 13). 反应的底物普适性较好, 不受苯 乙烯类底物的取代基位置和电子性质的影响，都能以良 好的产率得到相应环丙烷类化合物. 有趣的是，铁、铜、 钉以及 $\operatorname{Co}(s a l e n)$ 络合物等典型的环丙烷化催化剂并不 能很好地催化该反应.

2017 年, 该团队 ${ }^{[37] 又 以 ~} \alpha, \beta$-不饱和砜 39 为底物, 系 统研究了不同氟烷基重氮化合物应用于 $[3+2]$ 环加成反 应的差异，并开发了安全、简便合成砜基、氟烷基双取 代的吡唑啉类化合物 40 的新方法 (Scheme 14). 与此前 报道的一步合成二氟甲基取代环丙烷的反应情况类

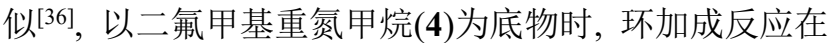
相转移条件下或原位生成二氟甲基重氮甲烷的条件下 不发生或反应性极差，在连续流反应条件下却以优秀的 产率获得相应的产物并实现了克级制备.

2017 年, Britton 和 Jamison ${ }^{[38]}$ 采用多步连续流动新 策略，高效、高产率地合成了含氟吡唑类化合物和吡唑 啉类化合物, 并通过添加反应模块对其进行一系列衍生 反应，如 $N$-烷基化和芳基化、脱保护基及酰胺化反应等, 多样化地构筑了目标分子(Scheme 15). 连续流反应器 


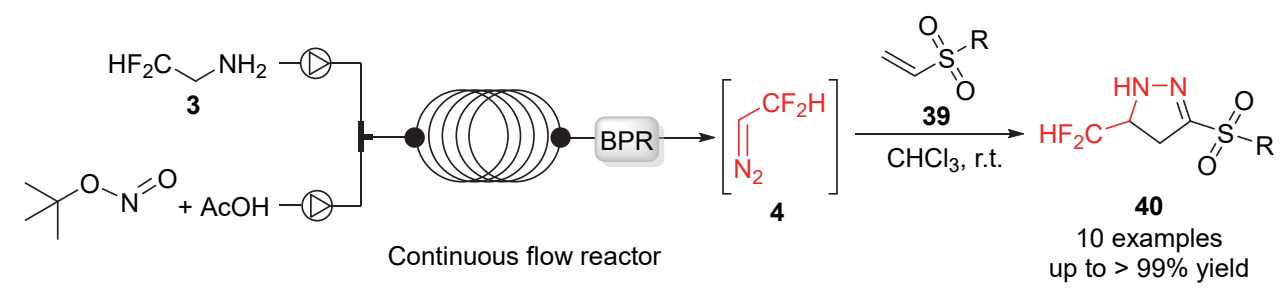

图式 14 采用连续流反应器的二氟甲基重氮甲烷的 $[3+2]$ 环加成反应

Scheme $14[3+2]$ cycloaddition reaction of $\mathrm{CF}_{2} \mathrm{HCHN}_{2}$ using continuous flow reactors

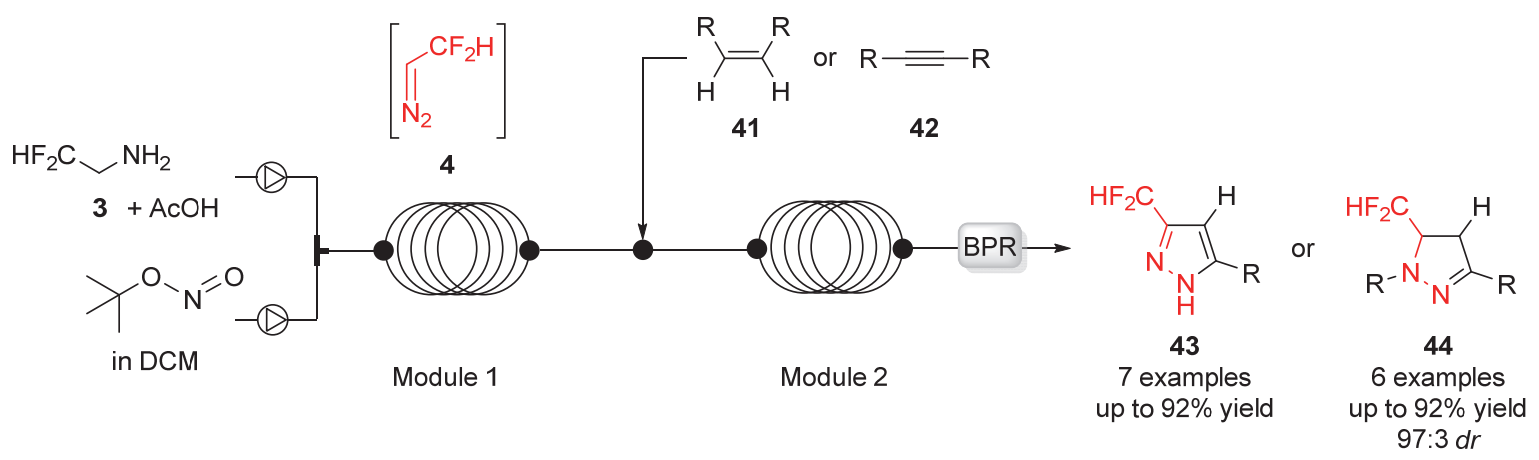

图式 15 多步连续流动合成含氟吡唑类和吡唑啉类化合物

Scheme 15 Multi-step continuous flow synthesis of fluorinated pyrazoles and pyrazolines

的使用使得该类反应可以在高于溶剂沸点的温度下进 行, 有效改善了反应动力学. 与传统方法相比, 该策略 极大地缩短了反应时间且无需使用催化剂和碱. 值得一 提的是, 基于此连续流平台, 他们已成功合成了杀菌剂 联苯吡菌胺和氟唑菌酰胺(图 4) ${ }^{[39]}$.<smiles>Cn1cc(C(=O)Nc2ccc(F)cc2-c2ccc(Cl)c(Cl)c2)c(C(F)(F)F)n1</smiles>

Bixafen $38 \%$ yield

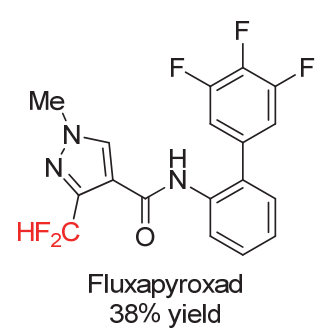

图 4 基于连续流模块合成的含二氟甲基农药分子

Figure $4 \mathrm{CF}_{2} \mathrm{H}$-containing pesticides synthesized by continuous flow modules

\section{6 二氟甲基重氮甲烷的稳定替代物}

尽管连续流方法解决了二氟甲基重氮甲烷(4)在反 应过程中存在的安全风险, 但它仍然存在操作繁琐及成 本高等不足. 为扩大二氟甲基重氮甲烷(4)在有机合成 及药物化学等领域的应用范围, 研究学者们进一步开发 了一些新型的、稳定的二氟甲基化试剂用于替代二氟甲 基重氮甲烷.

除重氮化合物外, 硫叶立德也是一类重要的卡宾前 体. 基于此, 肖吉昌研究小组于 2017 年开发了一种硫叶
立德型二氟甲基试剂: $\mathrm{Ph}_{2} \mathrm{~S}^{+} \mathrm{CH}_{2} \mathrm{CF}_{2} \mathrm{H}^{-} \mathrm{OTf}$ (47). 该试剂 常态下为固体，易于制备、保存和称量，在碱促进和铁 催化的作用下即可原位生成 $\mathrm{Fe}$-二氟甲基卡宾络合物. 他们以 $\mathrm{FeTPPCl}$ 为催化剂, 在还原剂的存在下, 高产率 地实现了 47 与烯烃 48 的环丙烷化反应 $(\text { Scheme } 16, a)^{[40]}$ 以及与硅烷 50 的 $\mathrm{Si}-\mathrm{H}$ 键的插入反应 $(\text { Scheme 16, b })^{[41]}$. 需要注意的是, 锌粉在反应中起到了至关重要的作用: (1)由于 47 还原性较弱, 反应中需添加强还原剂锌粉使 $\mathrm{Fe}(\mathrm{III})$ 还原为 $\mathrm{Fe}(\mathrm{II})$; (2)过量的锌粉会分解 47, 降低产 率. 该团队 ${ }^{[42] 于 ~} 2018$ 年又报道了可见光催化的芳基烯 烃 52 与 47 的二氟烷基化反应，通过改变溶剂实现了芳 基烯烃的二氟乙基化及双官能化反应(Scheme 16, c).

2018 年, 马军安课题组 ${ }^{[43]}$ 以溴二氟乙酸乙酯为原 料, 经硫化、酯还原、硫化物氧化、胺化及重氮化五步 反应制备了一种新型的二氟甲基化试剂一苯砜基二氟 重氮乙烷 Ps-DFA (56). 该物质在常温常压下为液体且 结构稳定, 作为含二氟甲基砌块与缺电子烯烃和炔烃均 能发生 $[3+2]$ 环加成反应(Scheme 17, a). 其中, 与炔烃 反应所得产物 58 可在镁粉的作用下脱去砜基, 转化为 二氟甲基取代的吡唑类化合物 57. 在此工作的基础上, 该研究团队 [44]于 2019 年报道了银催化 Ps-DFA 与芳基重 氮盐的区域选择性 $[3+2]$ 环加成反应，并利用该方法学 快速合成了 P 2 X3 受体拮抗剂的二氟甲基类似物 (Scheme 17, b). 该反应条件温和, 底物普适性好, 以高 达 95\%的收率得到了一系列四氮唑类化合物 60 . 同年, 


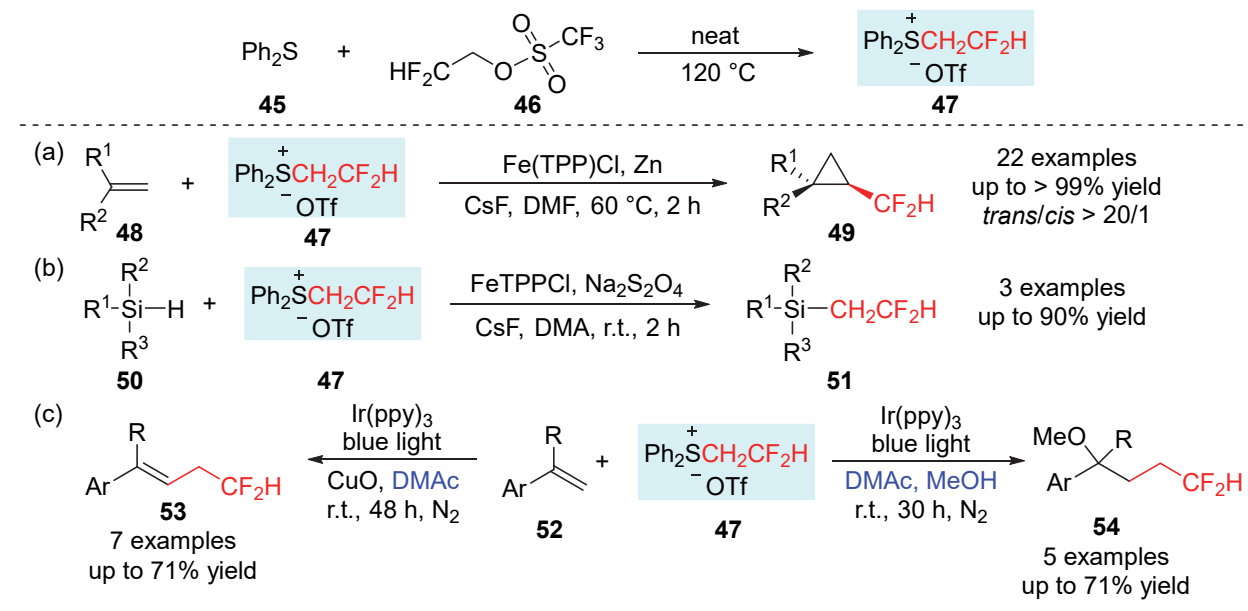

图式 $16 \mathrm{Ph}_{2} \mathrm{~S}^{+} \mathrm{CH}_{2} \mathrm{CF}_{2} \mathrm{H}^{-} \mathrm{OTf}$ 作为二氟甲基重氮甲烷替代物参与的反应

Scheme 16 Reactions of $\mathrm{Ph}_{2} \mathrm{~S}^{+} \mathrm{CH}_{2} \mathrm{CF}_{2} \mathrm{H}^{-} \mathrm{OTf}$ as the surrogate of $\mathrm{CF}_{2} \mathrm{HCHN}_{2}$

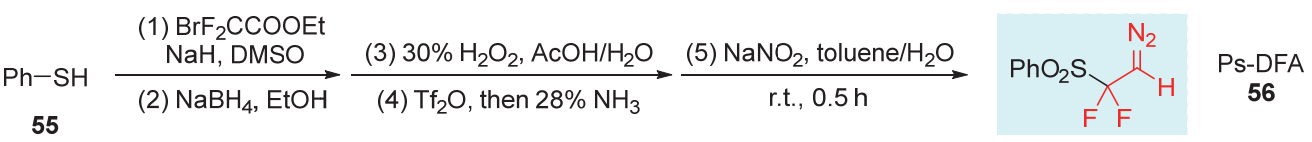

(a)

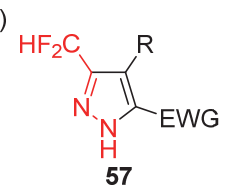

12 examples

up to $92 \%$ yield

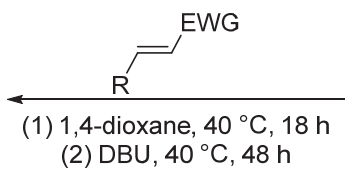

(2) $\mathrm{DBU}, 40^{\circ} \mathrm{C}, 48 \mathrm{~h}$

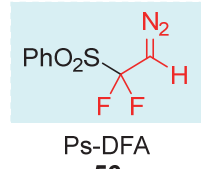

56

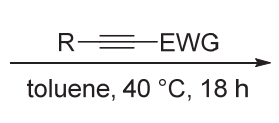

$\mathrm{PhO}_{2} \mathrm{SF}_{2} \mathrm{C}$

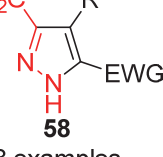

8 examples up to $99 \%$ yield

(b)

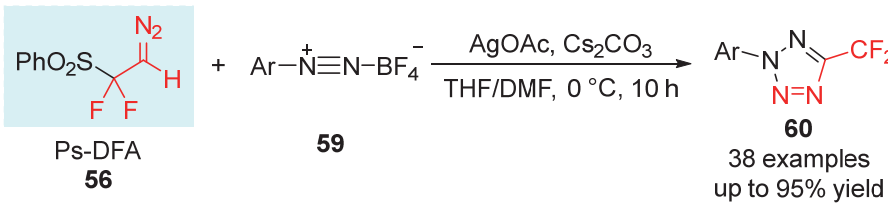

(c)

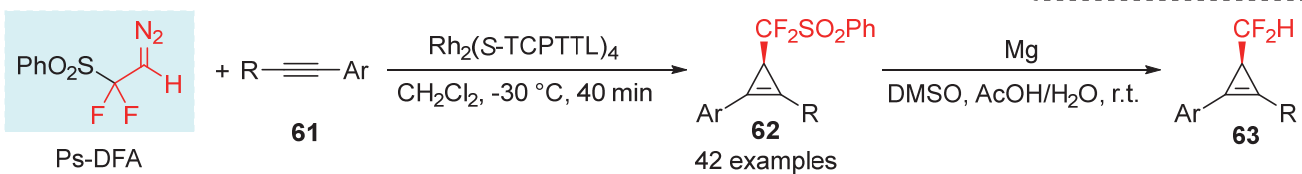

up to $99 \%$ yield, up to $97 \%$ ee

(d)<smiles>COc1ccc(N)cc1</smiles>

(e)

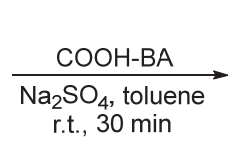

65

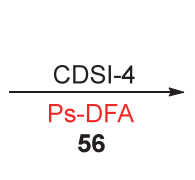

up to $70 \%$ yield, $73 \%$ ee, $>50: 1 d r$
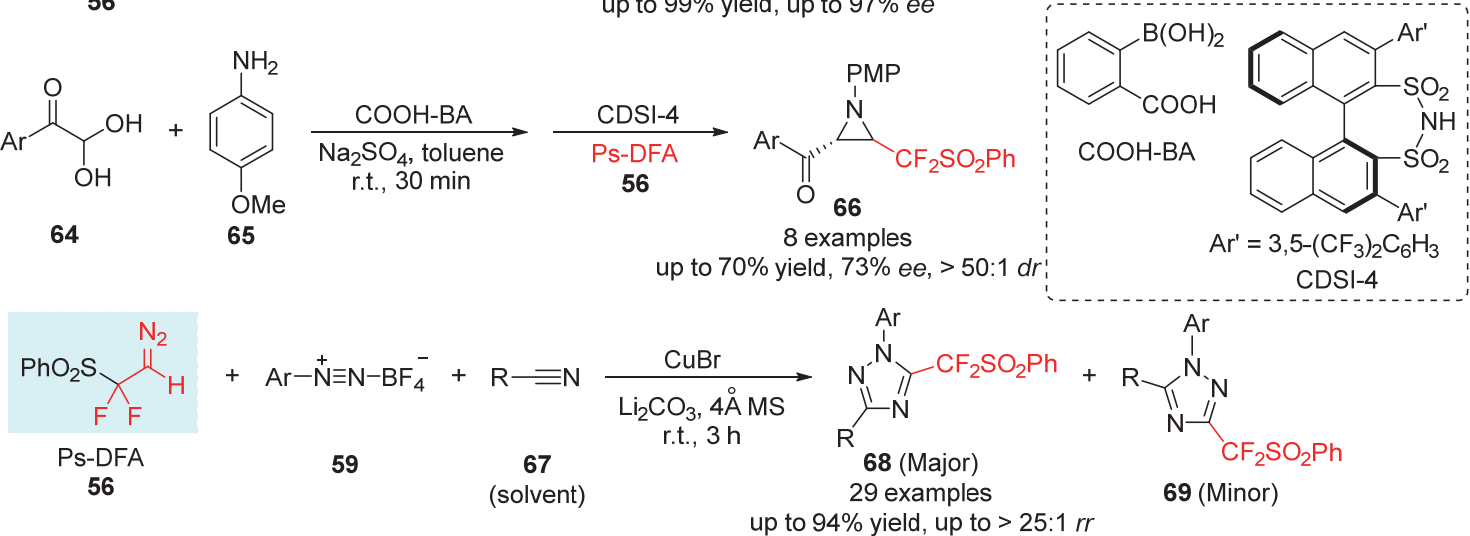

图式 17 Ps-DFA 作为二氟甲基重氮甲烷替代物参与的反应

Scheme 17 Reactions of Ps-DFA as the surrogate of $\mathrm{CF}_{2} \mathrm{HCHN}_{2}$ 
马军安课题组 ${ }^{[45]}$ 还报道了手性 Rh(II)催化的 Ps-DFA 与 一系列内炔的高度对映选择性环丙烯反应, 该反应是首 例催化仅含有电子受体的卡宾前体与内炔的不对称加 成反应(Scheme 17, c). 2020 年, 该研究团队 ${ }^{[46]}$ 进一步研 究了手性 Brønsted 酸催化的 Ps-DFA 与原位生成的醛亚 胺的不对称吅啶化反应(Scheme 17,d). 尽管得到的产 物对映选择性不高, 但经过简单的溶解-过滤操作后, 部分产物几乎可以达到光学纯. 同年, 马军安课题组 ${ }^{[47]}$ 通过铜催化的 Ps-DFA (56) 与芳基重氮盐 59 和腈 67 的 $[1+2+2]$ 环加成反应合成了二氟甲基取代三氮唑化合 物 68 (Scheme 17, e). 值得一提的是, 三氟甲基重氮甲 烷(2)在相同条件下也适用于该三组分反应.

目前, 二氟甲基重氮甲烷(4)仅通过原位 ${ }^{[19]}$ 和非原 位 ${ }^{[29]}$ 方式制备, 且由于反应过程中迅速生成的大量二 氟甲基重氮甲烷(4)会积聚于反应器中, 该制备方法存 在着一定的安全隐患. 2020 年, 毕锡和团队 ${ }^{[48]}$ 设计并合 成的二氟乙醛 $N$-邻三氟甲基苯磺酰腙(71, DFHZ-Tfs) 在 很大程度上解决了二氟甲基重氮甲烷(4)的安全生产及 使用问题. DFHZ-Tfs (71)的制备方法高效且实用, 合成 原料为商业易得的二氟乙酸乙酯. 且 71 性质稳定, 常态 下为结晶固体, 不易分解, 方便存储和运输, 在碱的作 用下即可原位生成二氟甲基重氮甲烷(4). 需要注意的 是, 该过程需要在无水条件下进行, 水的存在会使得二 氟甲基重氮甲烷(4)转化为甲酰基重氮甲烷. DFT 理论计 算表明, 发生这种转化的原因是碱 $(\mathrm{NaOH})$ 与水通过氢 键的协同作用促进了水合反应. 目前, DFHZ-Tfs 已被应
用于与烯烃的环丙烷化反应以及 Doyle-Kirmse 反应，反 应产率高且选择性好(Scheme 18).

\section{7 总结与展望}

二氟甲基重氮甲烷作为含氟砌块通过不同类型的 反应实现了众多二氟甲基取代的有机化合物的构建. 目 前, 对其应用的报道以与烯烃和炔烃的 1,3-偶极环加成 反应居多, 使得产物结构类型较为单一. 因此, 需要进 一步探索二氟甲基重氮甲烷与不同类型底物(如醛、酮、 亚胺、联烯、烯酮、硝酮、芳基硼酸或酯、卤代烃等) 的反应并拓展其反应类型. 同时，随着二氟甲基化方法 的快速发展，非手性二氟甲基化合物的合成方法较为成 熟，但高效的不对称二氟甲基化反应体系的构建仍是亟 待解决的技术难题. 基于对二氟甲基重氮甲烷反应性的 认知及其类似物在不对称二氟甲基化反应中的应用，开 展其催化不对称研究具有重要意义, 尤其是二氟甲基重 氮甲烷直接参与的高对映选择性和非对映选择性合成 结构多样化的含二氟甲基杂环化合物的化学转化. 此 外，新型手性配体及手性催化剂的开发也将推动不对称 二氟甲基化反应体系的建立.二氟甲基重氮甲烷具有的 爆炸性、挥发性等缺点要求我们必须进一步研究其安全 使用方法，并发展更多新型、稳定、高效、绿色的替代 物。总而言之，二氟甲基重氮甲烷作为含氟砌块的应用 研究在 5 年间取得了一定的进展, 但仍具有较大研究潜 力和发展空间.

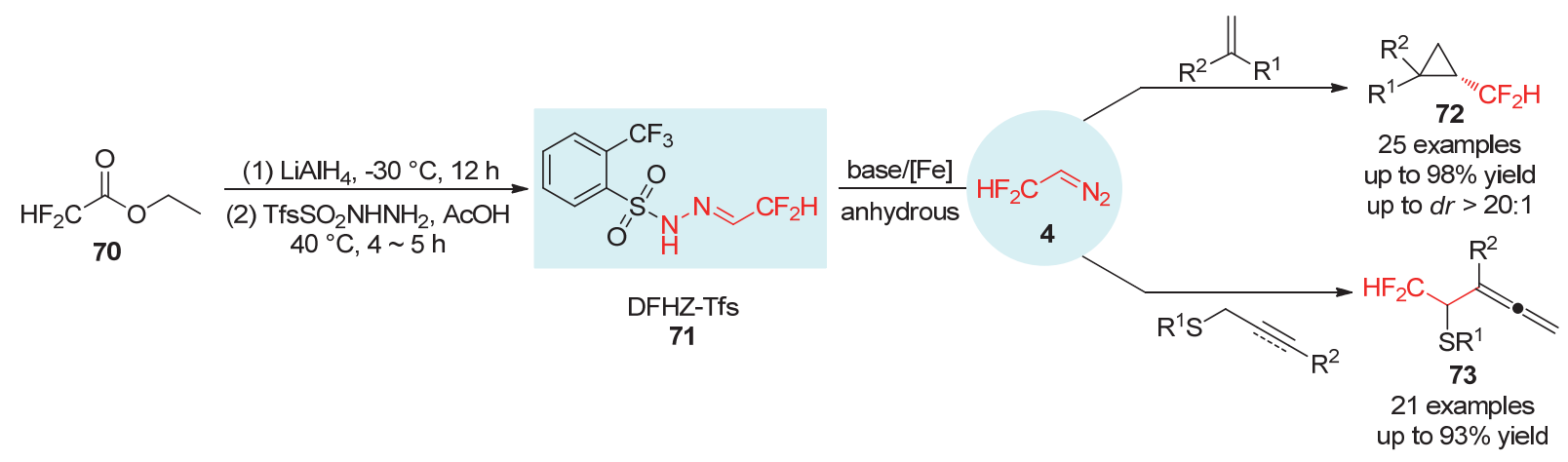

图式 18 DFHZ-Tfs 作为二氟甲基重氮甲烷替代物参与的反应

Scheme 18 Reactions of DFHZ-Tfs as the surrogate of $\mathrm{CF}_{2} \mathrm{HCHN}_{2}$

\section{References}

[1] (a) Gouverneur, V.; Muller, K. Fluorine in Pharmaceutical and Medicinal Chemistry: From Biophysical Aspects to Clinical Applications, Im-perial College Press, London, 2012.

(b) Zhang, J.; Jin, C.; Zhang, Y. Chin. J. Org. Chem. 2014, 34, 662 (in Chinese).

(张雯, 金传飞, 张英俊, 有机化学, 2014, 34, 662.)

(c) Xing, L.; Blakemore, D. C.; Narayanan, A.; Unwalla, R.; Lovering, F.; Denny, R. A.; Zhou, H.; Bunnage, M. E. ChemMedChem
2015, 10, 715 .

(d) Mei, H.; Han, J.; Fustero, S.; Medio-Simon, M.; Sedgwick, D. M.; Santi, C.; Ruzziconi, R.; Soloshonok, V. A. Chem.-Eur. J. 2019, 25,11797

[2] (a) Meanwell, N. A. J. Med. Chem. 2011, 54, 2529.

(b) Zafrani, Y.; Sod-Moriah, G.; Yeffet, D.; Berliner, A.; Amir, D.; Marciano, D.; Elias, S.; Katalan, S.; Ashkenazi, N.; Madmon, M.; Gershonov, E.; Saphier, S. J. Med. Chem. 2019, 62, 5628.

[3] (a) Mori, T.; Ujihara, K.; Matsumoto, O.; Yanagi, K.; Matsuo, N. J. Fluorine Chem. 2007, 128, 1174. 
(b) Thappali, S. R.; Varanasi, K. V.; Veeraraghavan, S.; Vakkalanka, S. K.; Mukkanti, K. J. Mass Spectrom. 2012, 47, 1612.

(c) Giornal, F.; Pazenok, S.; Rodefeld, L.; Lui, N.; Vors, J.-P.; Leroux, F. R. J. Fluorine Chem. 2013, 152, 2.

(d) Rodriguez-Torres, M.; Glass, S.; Hill, J.; Freilich, B.; Hassman, D.; Di Bisceglie, A. M.; Taylor, J. G.; Kirby, B. J.; Dvory-Sobol, H.; Yang, J.-C.; An, D.; Stamm, L. M.; Brainard, D. M.; Kim, S.; Krefetz, D.; Smith, W.; Marbury, T.; Lawitz, E. J. Viral Hepatitis 2016, 23, 614

(e) Lamb, Y. N. Drugs 2017, 77, 1797.

(f) Zeng, J.; Xu, Z.; Ma, J. Chin. J. Org. Chem. 2020, 40, 1105 (in Chinese).

(曾俊良, 许志红, 马军安, 有机化学, 2020, 40, 1105.)

[4] For selected reviews on difluoromethylation, see: (a) Hu, J.; Zhang, W.; Wang, F. Chem. Commun. 2009, 7465.

(b) Lu, Y.; Liu, C.; Chen, Q.-Y. Curr. Org. Chem. 2015, 19, 1638.

(c) Belhomme, M. C.; Besset, T.; Poisson, T.; Pannecoucke, X. Chem.-Eur. J. 2015, 21, 12836.

(d) Ni, C.; Zhu, L.; Hu, J. Acta Chim. Sinica 2015, 73, 90 (in Chinese).

(倪传法, 朱林桂, 胡金波, 化学学报, 2015, 73, 90.)

(e) Xu, P.; Guo, S.; Wang, L.; Tang, P. Synlett 2015, 26, 36.

(f) Rong, J.; Ni, C.; Hu, J. Asian J. Org. Chem. 2017, 6, 139.

(g) Yerien, D. E.; Barata-Vallejo, S.; Postigo, A. Chem.-Eur. J. 2017, 23, 14676.

(h) Wang, W.; Yu, Q.; Zhang, Q.; Li, J.; Hui, F.; Yang, J.; Lü, J.; Jin, C.; Zhang, Y. Chin. J. Org. Chem. 2018, 38, 1569 (in Chinese). (王为强, 余秦伟, 张前, 李江伟, 惠丰, 杨建明, 吕剑, 金传飞, 张英俊, 有机化学, 2018, 38, 1569.)

(i) Feng, Z.; Xiao, Y.-L.; Zhang, X. Acc. Chem. Res. 2018, 51, 2264.

(j) Mykhailiuk, P. K.; Koenigs, R. M. Chem.-Eur. J. 2019, 25, 6053.

[5] Deng, X.-Y.; Lin, J.-H.; Zheng, J.; Xiao, J.-C. Chem. Commun. 2015, 51, 8805 .

[6] Li, L.; Wang, F.; Ni, C.; Hu, J. Angew. Chem., Int. Ed. 2013, 52, 12390.

[7] For a recent example, see: Gao, X.; He, X.; Zhang, X. Chin. J. Org. Chem. 2019, 39, 215 (in Chinese).

(高兴, 何旭, 张新刚, 有机化学, 2019, 39, 215.)

[8] Zhao, Y.; Huang, W.; Zheng, J.; Hu, J. Org. Lett. 2011, 13, 5342.

[9] For a review, see: Tao, X.; Sheng, R.; Bao, K.; Wang, Y.; Jin, Y. Chin. J. Org. Chem. 2019, 39, 2726 (in Chinese).

(陶雪芬, 盛荣, 鲍斿, 王玉新, 金银秀, 有机化学, 2019，39, 2726.)

[10] Xu, L.; Vicic, D. A. J. Am. Chem. Soc. 2016, 138, 2536.

[11] Chang, D.; Gu, Y.; Shen, Q. Chem.-Eur. J. 2015, 21, 6074.

[12] Fujita, T.; Sanada, S.; Chiba, Y.; Sugiyama, K.; Ichikawa, J. Org. Lett. 2014, 16, 1398.

[13] Hanamoto, T.; Kurosato, F.; Ishikawa, T.; Yamada, Y. Synlett 2015, 26, 1827.

[14] Zafrani, Y.; Sod-Moriah, G.; Segall, Y. Tetrahedron 2009, 65, 5278.

[15] For selected reviews, see: (a) Zhang, Y.; Wang, J. Chem. Commun. 2009, 36, 5350 .

(b) Xiao, Q.; Zhang, Y.; Wang, J. Acc. Chem. Res. 2013, 46, 236.

(c) Qiu, D.; Qiu, M.; Ma, R.; Zhang, Y.; Wang, J. Acta Chim. Sinica 2016, 74, 472 (in Chinese).

(邱頔, 邱孟龙, 马戎, 张艳, 王剑波, 化学学报, 2016, 74, 472.)

(d) Liu, L.; Zhang, J. Chin. J. Org. Chem. 2017, 37, 1117 (in Chinese).

(刘路, 张俊良, 有机化学, 2017, 37, 1117.)

(e) Gao, Y.; Wang, J. Chin. J. Org. Chem. 2018, 38, 1275 (in Chi- nese).

(趴云鹏，王剑波，有机化学, 2018, 38, 1275.)

(f) Yang, Z.; Stivanin, M. L.; Jurberg, I. D.; Koenigs, R. M. Chem. Soc. Rev. 2020, 49, 6833 .

(g) Zhao, R.; Shi, L. Angew. Chem., Int. Ed. 2020, 59, 12282.

(h) Wu, S.; Song, H.-X.; Zhang, C.-P. Chem.-Asian J. 2020, 15, 1660.

[16] Gilman, H.; Jones, R. G. J. Am. Chem. Soc. 1943, 65, 1458.

[17] For a leading review, see: (a) Mykhailiuk, P. K. Chem Rev. 2020, $120,12718$.

For selected references, see: (b) Morandi, B.; Mariampillai, B.; Carreira, E. M. Angew. Chem., Int. Ed. 2011, 50, 1101.

(c) Wu, G.; Deng, Y.; Wu, C.; Wang, X.; Zhang, Y.; Wang, J. Eur. J. Org. Chem. 2014, 2014, 4477.

(d) Luo, H.; Wu, G.; Zhang, Y.; Wang, J. Angew. Chem., Int. Ed. 2015, 54, 14503.

(e) Chen, Z.; Zheng, Y.; Ma, J.-A. Angew. Chem., Int. Ed. 2017, 56, 4569.

(f) Chen, Z.; Ren, N.; Ma, X.; Nie, J.; Zhang, F.-G.; Ma, J.-A. ACS Catal. 2019, 9, 4600 .

(g) Li, J.; Zhang, D.; Chen, J.; Ma, C.; Hu, W. ACS Catal. 2020, 10, 4559.

[18] (a) Atherton, J. H.; Fields, R.; Haszeldine, R. N. J. Chem. Soc. C 1971, 366

(b) Mykhailiuk, P. K. Org. Biomol. Chem. 2015, 13, 3438.

[19] Mykhailiuk, P. K. Angew. Chem., Int. Ed. 2015, 54, 6558.

[20] Li, J.; Yu, X.-L.; Cossy, J.; Lv, S.-Y.; Zhang, H.-L.; Su, F.; Mykhailiuk, P. K.; Wu, Y. Eur. J. Org. Chem. 2017, 2017, 266.

[21] Zheng, Y.; Yu, X.; Lv, S.; Mykhailiuk, P. K.; Ma, Q.; Hai, L.; Wu, Y. RSC Adv. 2018, 8, 5114.

[22] Lebed, P. S.; Fenneteau, J.; Wu, Y.; Cossy, J.; Mykhailiuk, P. K. Eur. J. Org. Chem. 2017, 2017, 6114.

[23] Feraldi-Xypolia, A.; Fredj, G.; Tran, G.; Tsuchiya, T.; Vors, J.-P.; Mykhailiuk, P.; GomezPardo, D.; Cossy, J. Asian J. Org. Chem. 2017, 6, 927 .

[24] Han, W.-Y.; Zhao, J.; Wang, J.-S.; Xiang, G.-Y.; Zhang, D.-L.; Bai, M.; Cui, B.-D.; Wan, N.-W.; Chen, Y.-Z. Org. Biomol. Chem. 2017, $15,5571$.

[25] Han, W.-Y.; Zhao, J.; Wang, J.-S.; Cui, B.-D.; Wan, N.-W.; Chen, Y.-Z. Tetrahedron 2017, 73, 5806.

[26] Han, W.-Y.; Zhao, J.; Cui, B.-D.; Chen, Y.-Z. Chin. J. Synth. Chem. 2018, 26, 328 (in Chinese).

(韩文勇, 赵佳, 崔宝东, 陈永正, 合成化学, 2018, 26, 328.)

[27] Han, W.-Y.; Wang, J.-S.; Zhao, J.; Long, L.; Cui, B.-D.; Wan, N.-W.; Chen, Y.-Z. J. Org. Chem. 2018, 83, 6556.

[28] Wang, J.-S.; Shan, J.; Bai, M.; Cui, B.-D.; Wan, N.-W.; Wang, Y.-S.; Han, W.-Y.; Chen, Y.-Z. Tetrahedron 2018, 74, 3904.

[29] Wang, J.-S.; Huang, K.-S.; Han, W.-Y.; Cui, B.-D.; Wan, N.-W.; Chen, Y.-Z. Org. Lett. 2019, 21, 8751.

[30] Zhang, X.-W.; Hu, W.-L.; Chen, S.; Hu, X.-G. Org. Lett. 2018, 20, 860.

[31] Gao, Y.; Peng, S.-Q.; Liu, D.-Y.; Rui, P.-X.; Hu, X.-G. Eur. J. Org. Chem. 2019, 2019, 1715 .

[32] Mykhailiuk, P. K.; Kishko, I.; Kubyshkin, V.; Budisa, N.; Cossy, J. Chem.-Eur. J. 2017, 23, 13279.

[33] Peng, S.-Q.; Zhang, X.-W.; Zhang, L.; Hu, X.-G. Org. Lett. 2017, 19, 5689.

[34] Li, J.; Ma, C.; Xing, D.; Hu, W. Org. Lett. 2019, 21, 2101.

[35] Mertens, L.; Hock, K. J.; Koenigs, R. M. Chem.-Eur. J. 2016, 22, 9542.

[36] Hock, K. J.; Mertens, L.; Koenigs, R. M. Chem. Commun. 2016, $52,13783$. 
[37] Hock, K. J.; Mertens, L.; Metze, F. K.; Schmittmann, C.; Koenigs, R. M. Green Chem. 2017, 19, 905.

[38] Britton, J.; Jamison, T. F. Angew. Chem., Int. Ed. 2017, 56, 8823.

[39] Britton, J.; Jamison, T. F. Eur. J. Org. Chem. 2017, 2017, 6566.

[40] Duan, Y.; Lin, J.-H.; Xiao, J.-C.; Gu, Y.-C. Chem. Commun. 2017, 53, 3870.

[41] Duan, Y.; Lin, J.-H.; Xiao, J.-C.; Gu, Y.-C. Org. Chem. Front. 2017, 4, 1917.

[42] Pan, X.-Y.; Zhao, Y.; Qu, H.-A.; Lin, J.-H.; Hang, X.-C.; Xiao, J.-C. Org. Chem. Front. 2018, 5, 1452.

[43] Zeng, J.-L.; Chen, Z.; Zhang, F.-G.; Ma, J.-A. Org. Lett. 2018, 20, 4562 .
[44] Peng, X.; Xiao, M.-Y.; Zeng, J.-L.; Zhang, F.-G.; Ma, J.-A. Org. Lett. 2019, 21, 4808.

[45] Zhang, Z.-Q.; Zheng, M.-M.; Xue, X.-S.; Marek, I.; Zhang, F.-G.; Ma, J.-A. Angew. Chem., Int. Ed. 2019, 58, 18191.

[46] Tan, X.-F.; Zhang, F.-G.; Ma, J.-A. Beilstein J. Org. Chem. 2020, $16,638$.

[47] Peng, X.; Zhang, F.-G.; Ma, J.-A. Adv. Synth. Catal. 2020, 362, 4432.

[48] Ning, Y.; Zhang, X.; Gai, Y.; Dong, Y.; Sivaguru, P.; Wang, Y.; Reddy, B. R. P.; Zanoni, G.; Bi, X. Angew. Chem., Int. Ed. 2020, 59,6473 .

(Cheng, F.) 